\title{
Continuous Electroencephalography Monitoring in Adults in the Intensive Care Unit
}

\author{
Anselmo Caricato ${ }^{*}$, Isabella Melchionda and Massimo Antonelli
}

\begin{abstract}
This article is one of ten reviews selected from the Annual Update in Intensive Care and Emergency Medicine 2018. Other selected articles can be found online at https://www.biomedcentral.com/collections/ annualupdate2018. Further information about the Annual Update in Intensive Care and Emergency Medicine is available from http://www.springer.com/series/8901.
\end{abstract}

\section{Background}

Neurologic monitoring in the intensive care unit (ICU) is based on the acquisition of several parameters from multiple brain devices. Electroencephalography (EEG) is one of the simplest ways to investigate cerebral activity, easily recorded at the bedside and sensitive to changes in both brain structure and function [1]. Due to these features and its simple utilization, use of continuous EEG (cEEG) recording in critically ill patients has increased over the past decade [2,3]. Recently, it was recommended by international guidelines with well-defined indications [4-6]. Nonetheless, few academic centers use it, and the expertise of neurointensivists for continuous real-time interpretation of EEG patterns is uncommon [7].

This chapter summarizes recent results on this topic, focusing on indications, duration of monitoring and technical issues.

\section{Indications}

Non-convulsive Seizures and Non-convulsive Status Epilepticus

Recent classifications define convulsive status epilepticus as a condition of abnormally prolonged seizures lasting

\footnotetext{
* Correspondence: anselmo.caricato@unicatt.it

Università Cattolica del Sacro Cuore, Department of Anesthesiology and Intensive Care Medicine, Fondazione Policlinico Universitario A. Gemelli, Rome, Italy
}

more than $5 \mathrm{~min}$. Non-convulsive status epilepticus (NCSE) is defined as abnormally prolonged seizures without prominent motor symptoms. The limit for 'prolonged' remains to be determined: in selected cases of NCSE, for example, focal status epilecticus with impaired consciousness, it was defined as 10 min by a Task Force from the International League against Epilepsy [8].

Several authors have reported that NCSE is a common finding in critically ill patients, associated with increased mortality and increased risk of poor neurological outcome [9-11].

To exclude this condition, a consensus statement of the American Clinical Neurophysiology Society recommend use of cEEG [4]:

1. after seizures, if impaired consciousness persists after initial treatment

Evidence is strong for this recommendation. In ICU patients admitted for seizures, DeLorenzo et al. reported that non-convulsive seizures were observed on cEEG in $48 \%$ of cases, and NCSE in $14 \%$ of cases [12]. Claassen et al. identified non-convulsive seizures on cEEG monitoring in $25 \%$ of 570 critical care patients; in a subgroup of 110 patients who had seizures before monitoring, non-convulsive seizures were observed on cEEG in $43 \%$ of the cases [13]. Several authors have confirmed these results, providing solid arguments for extended EEG monitoring in this condition [4].

2. in cases of unexplained alteration of mental status without known acute brain injury

In one prospective study, $75 \%$ of diagnoses of NCSE or non-convulsive seizures on cEEG were not preceded by clinical seizures. In these cases, symptoms may be minor or absent, or include aphasia, confusion, agitation or behavioral abnormalities [13]. Neurological fluctuation is a 
typical finding, and may help in diagnosis. When coma is present, NCSE is observed in $0-14 \%$ of the cases $[14,15]$

3. When specific EEG patterns are recognized on routine recording: generalized periodic discharges (GPDs), lateralized periodic discharges (LPDs), or BIPDs (bilateral independent periodic discharges) (Fig. 1)

In a large retrospective multicenter study in 4722 critically ill patients, based on the Critical Care EEG Monitoring Research Consortium multicenter database, there was significant relationship of seizures with some periodic and rhythmic patterns: GPDs, LPDs and lateralized rhythmic delta activity (LRDA) [16]. In these cases, the incidence of seizures ranged from $50 \%$ and $88 \%$ on routine EEG [4]. In particular, data showed that most of the seizures were non-convulsive. A detailed EEG description using standardized nomenclature is of paramount importance for a correct interpretation of EEG tracings, and for seizure risk stratification. These results strongly support the use cEEG to detect seizure occurrence when these pattern are found.

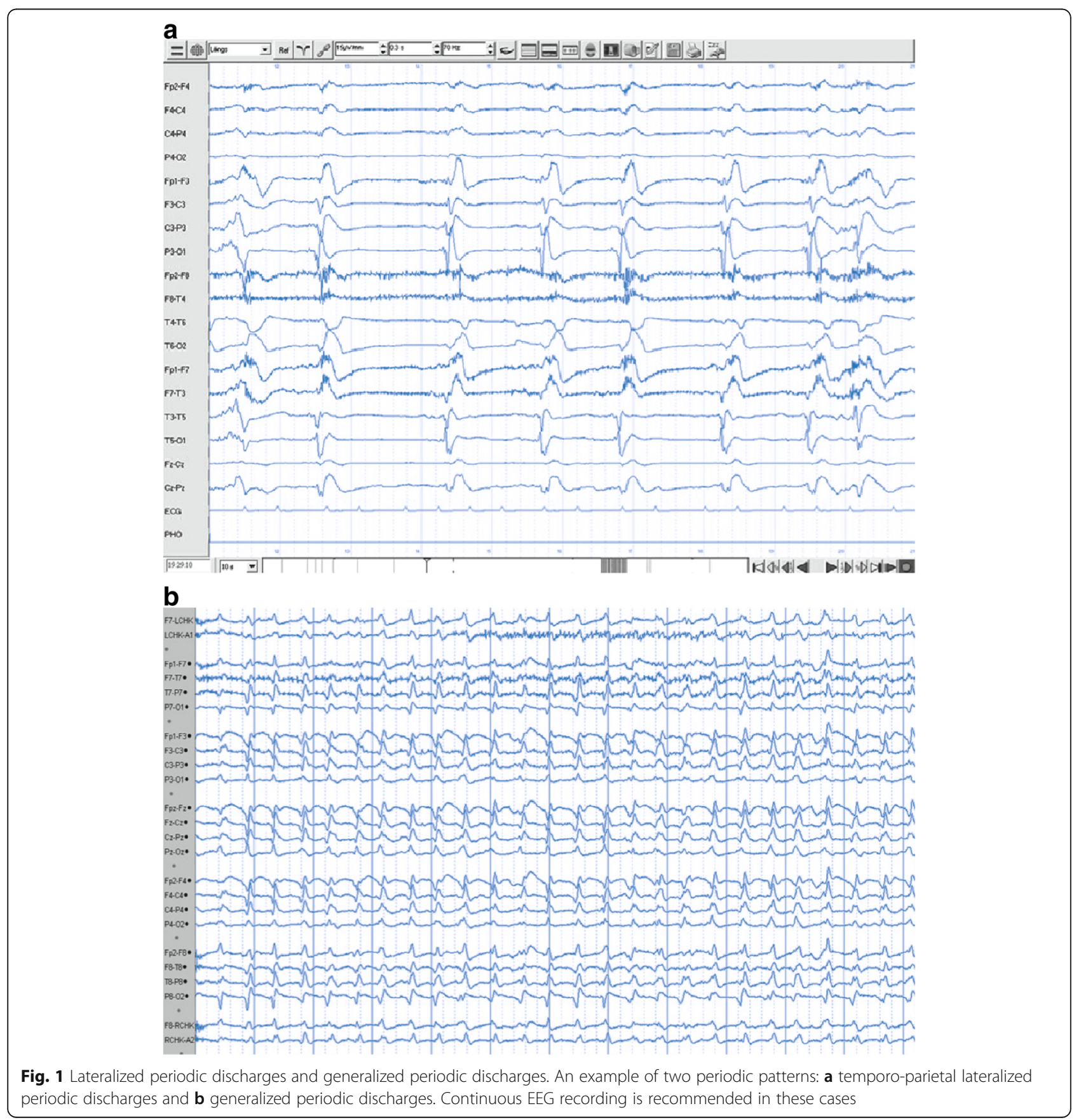




\section{In comatose patients after acute brain injury}

Several conditions have been reported to be correlated with NCSE, with incidences of up to $30 \%$ in subarachnoid hemorrhage, head injury, intracerebral hemorrhage, central nervous system infections [3]. The incidence may be even higher in post-anoxic encephalopathy, ranging from 10 to $60 \%$.

5. When neuromuscular blocking drugs (NMBD) are used in high risk patients

\section{Duration of CEEG Monitoring}

cEEG should be initiated as soon as possible when nonconvulsive seizures are suspected, as higher morbidity and mortality has been observed, and early treatments are likely to be more effective [17]. Recording for at least $24 \mathrm{~h}$ is recommended, but in some cases shorter or longer periods may be necessary. Traditional 30-60 min EEG recordings identify non-convulsive seizures in only $45-58 \%$ of patients in whom seizures are eventually recorded. About $80-95 \%$ of patients with non-convulsive seizures can be identified within 24-48 h $[18,19]$.

In 625 critically ill patients, the probability of seizures was strongly influenced by findings detected very early in the course of monitoring. In case of absence of epileptiform abnormalities during the first 15 min recording, the 72 -h seizure probability was less than $10 \%$, and less than $5 \%$ if no abnormality was present during the first 2 $\mathrm{h}$ recording [20].

In selected populations, the duration of monitoring is still a matter of debate. Thirty-minute serial EEGs have been demonstrated to have similar yield to cEEG in adult postcardiac arrest patients undergoing hypothermia [21]. Additional studies need to draw definitive data on this topic.

\section{Diagnosis of Ischemia}

EEG is very sensitive in detecting changes in cerebral blood flow (CBF), generally showing regional attenuation of faster frequencies and increases in slow activity; based on these observations, many authors have suggested cEEG for ischemia identification during surgical or neuroradiological procedures, during intracranial hypertension and for the diagnosis of delayed cerebral ischemia (DCI) after subarachnoid hemorrhage [22-24]. Although many studies have tried to show a specific ischemic pattern in various conditions, current recommendations suggest cEEG to detect ischemia only for the diagnosis of DCI after subarachnoid hemorrhage [5].

\section{Delayed Cerebral Ischemia}

DCI is a severe complication that occurs after subarachnoid hemorrhage in $20-30 \%$ of cases. Diagnosis is based on clinical symptoms and is characterized by a decrease in consciousness, new focal deficit, or both. No radiological examination can confirm or exclude DCI at the time of clinical deterioration; theoretically EEG is able to detect electrical changes before structural damage occurs, providing a window of opportunity for therapeutic interventions [25].

Several authors have investigated this hypothesis in small prospective studies using cEEG, and many quantitative EEG parameters have been evaluated. According to Claassen et al., a 10\% reduction in the alpha-delta power ratio had a sensitivity of $100 \%$ for $\mathrm{DCI}$, with a specificity of $76 \%$ [26]. Rots et al. confirmed these results in 20 patients with subarachnoid hemorrhage, reporting that a reduction in the alpha-delta ratio over time in any channel was the single most accurate prognostic indicator; patients in the DCI group showed a median decrease of $62 \%$ in the ratio, compared with a median increase of $27 \%$ in the control group [27]. Changes in alpha variability were also associated with DCI in these patients.

According to Rots et al. [27] and Gollwitzer et al. [28], EEG changes appearing before ischemia were identified in around $70 \%$ of cases; the median time between quantitative EEG (qEEG) changes and the clinical diagnosis of DCI ranged from 5 to $60 \mathrm{~h}$. At the Massachusetts General Hospital, an institutional protocol for patients with subarachnoid hemorrhage included cEEG recording, and prediction of DCI was suggested if there was an alpha-delta ratio decrease of $10 \%$ lasting $6 \mathrm{~h}(100 \%$ sensitivity and $76 \%$ specificity) or when at least $50 \%$ decrements in the alpha-delta ratio were observed for $2 \mathrm{~h}$ or more ( $89 \%$ sensitivity and $84 \%$ specificity) [29].

Recent international recommendations suggest use of cEEG to detect DCI in conjunction with other conventional examinations in comatose patients, in whom neurological examination is unreliable, and suggest that it may predict DCI 24-48 h prior to other diagnostic tools [5].

\section{Prognostication}

Some of the data obtained from EEG, such as sleep architecture, the presence of a sleep/awake cycle, reactivity to external stimuli, and detection of epileptiform changes can be used for prognostic evaluation in comatose patients [30]. In this setting, postanoxic encephalopathy has been extensively studied, and selected EEG patterns were found to be associated with a poor neurological outcome. A statement from the European Society of Intensive Care Medicine and the European Resuscitation Council suggests that the absence of EEG reactivity to external stimuli and the presence of burst-suppression or status epilepticus at $72 \mathrm{~h}$ after cardiac arrest significantly predict poor outcome, defined as severe neurological disability, persistent vegetative state or death, with a false positive ratio ranging from 0 to $6 \%$ [31].

Recently, Westhall et al. reported that the presence of a highly malignant EEG pattern, defined according to 
the EEG terminology standardized by the American Clinical Neurophysiology Society as suppressed background without discharges, suppressed background with continuous periodic discharges, or burst-suppression background with or without discharges, predicted poor outcome in comatose patients after cardiac arrest without false-positives [32].

Several studies have investigated the prognostic significance of EEG recordings in patients with subarachnoid hemorrhage $[9,10,26-28]$. Claassen et al. reported that outcome was poor (modified Rankin Scale 4 to 6) in all patients with absent EEG reactivity, GPDs, or BIPDs, and in $92 \%$ of patients with NCSE [33]. DeMarchis et al. confirmed these results, observing that patients with subarachnoid hemorrhage with seizures had more than 3-times higher odds of disability or death [10]. Whether cEEG adds information to serial EEG on this topic is not known. Duration of monitoring has not been investigated, and no definitive data can be drawn.

\section{Methods}

Based on the clinical scenario and available resources, different types of EEG monitoring can be chosen for recording in the ICU. It can be performed with portable EEG, mobile desktop EEG, and EEG workstations; in its most complete configuration, it is associated to a videocamera for a synchronous recording of video and EEG trace in order to minimize artifacts and recognize clinical correspondence with the EEG signal. Telemedicine programs may create connections between workstations remotely located in different places within or outside the hospital. This is of paramount importance, in particular when resources are lacking and neurologists and/or neurophysiologists are not always available.

\section{Electrode Positioning}

Most EEG recordings follow the American Clinical Neurophysiology Society International 10-20 system guidelines for head measurements and electrode applications [34]. The positions are determined as follows: reference points are the nasion, which is the depression at the top of the nose, at the eye level; and the inion, which is the bony lump at the base of the skull on the midline at the back of the head. From these points, the skull perimeters are measured in the transverse and median planes. The 10 and 20 refers to the electrode location, which is determined by dividing these perimeters into $10 \%$ and $20 \%$ intervals.

Different types of electrodes are available [35]. Disk or cup electrodes are classically used, and those with a central hole are best to permit periodic refilling with electrode conductive gel. When possible, computed tomography (CT) or magnetic resonance imaging (MRI) compatible electrodes should be used; they can remain in place during imaging, reducing time spent removing and reapplying, and may also reduce skin breakdown caused by frequent electrode removal and reapplication. Subdermal needle electrodes can be applied rapidly and do not require scalp abrasion. Needles are inserted just beneath the skin, parallel to the surface of the scalp and should be secured with adhesive paste. They are not recommended for awake patients or prolonged recording, but are very useful because of their rapid positioning and for brief recording in some comatose patients.

Subdermal wire electrodes are thin wires with a silver chloride tip, and are placed in a similar manner to that of the standard subdermal needle electrode. They are hooked into the lumen of a $25 \mathrm{G}$ or $27 \mathrm{G}$ needle; after positioning the needle is removed and the wire remains in the subdermal tissue. On the skin, some fixation with patches or glue may be useful. They may reduce skin breakdown and provide better recording performance compared to disc electrodes during prolonged cEEG monitoring [36]. An electrode cap and template system may be used when rapid initiation of EEG recording is essential. Their use may be limited by the presence of scalp wounds, dressings or intracranial devices (Fig. 2).

The number of electrodes used in cEEG studies varies considerably. Usually the 10-20 system places 21 electrodes. Alternatives to this full montage have been described. One of these is a sub-hairline recording, which uses four bipolar derivations: left temporal (left anterior temporal electrode to left mastoid electrode), left frontal (left frontal electrode to left anterior temporal electrode), right frontal (right frontal electrode to right anterior temporal electrode) and right temporal (right frontal anterior temporal electrode to right mastoid electrode) [37]. A montage with eight recording electrodes uses four couples of electrodes: forehead FP1, FP2; central C3, C4; temporal T3, T4; and occipital O1, O2. The reference electrodes should be at the bilateral earlobe or mastoid levels. The grounding electrode should be placed at the midpoint of the frontal pole $(\mathrm{FPz})$, and the common reference electrode should be placed at the median central point $(\mathrm{Cz})$ [37].

A full electrode configuration improves the ability to distinguish brain signals from artifact, aids in spatial localization of pathological activity, and provides redundancy, enabling continued interpretation in case one or more leads fail. However, in several cases, EEG recording is performed with a reduced number of electrodes, or in altered positions, in particular when surgical wounds, ventricular drains, or neuromonitoring devices limit the available surface of the skull. Under these conditions, electrode positioning is faster and easier to maintain. However, it is critical to maintain the symmetry of the left and right side electrodes.

For the application of long-term EEG monitoring, a 12-24 h suspension may be needed after $24-48 \mathrm{~h}$ of 

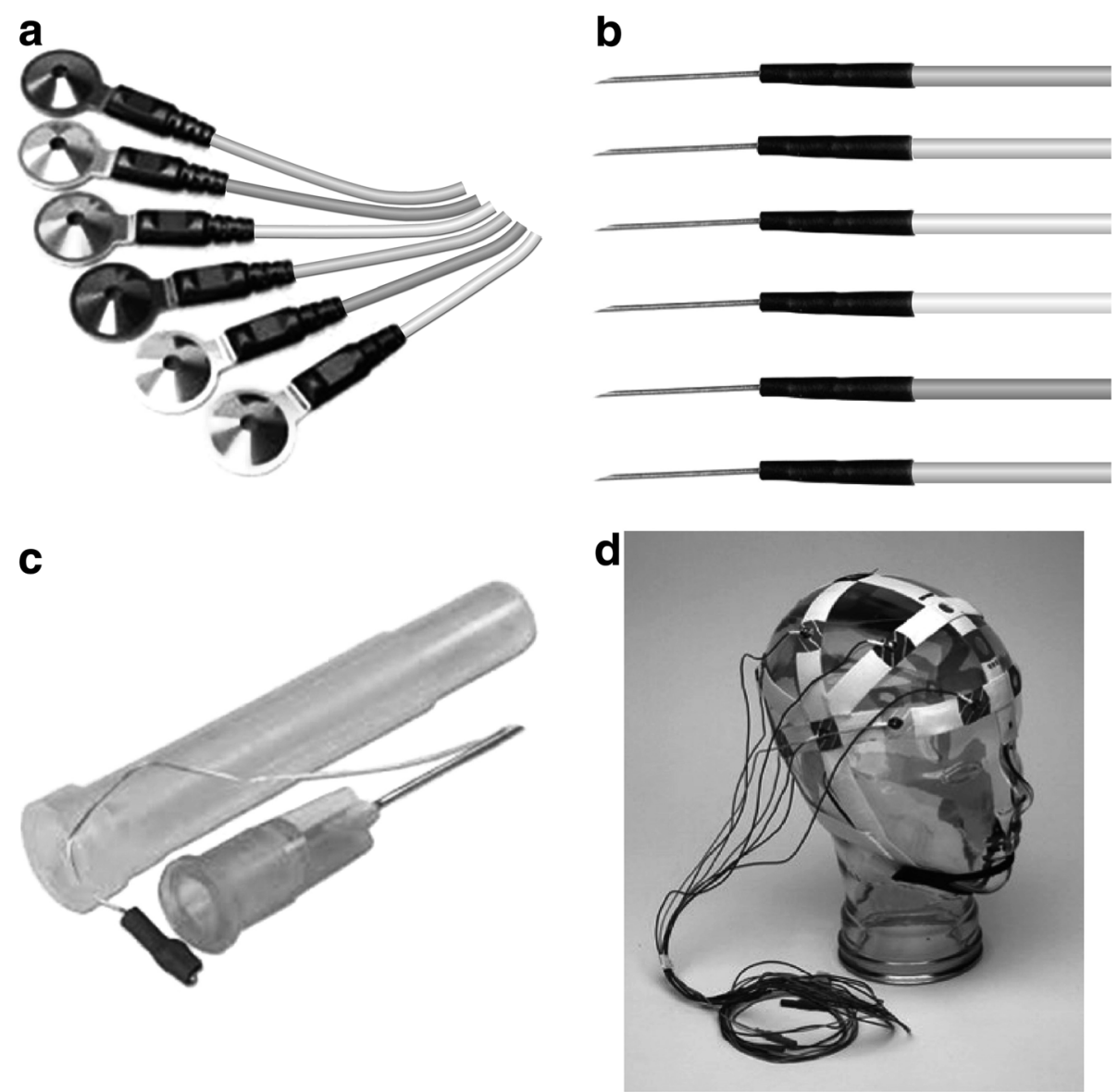

Fig. 2 Type of EEG electrodes. Examples of classic cup electrodes (a), subdermal needle electrodes (b), subdermal wire electrodes (c) and an EEG template system $(\mathbf{d})$

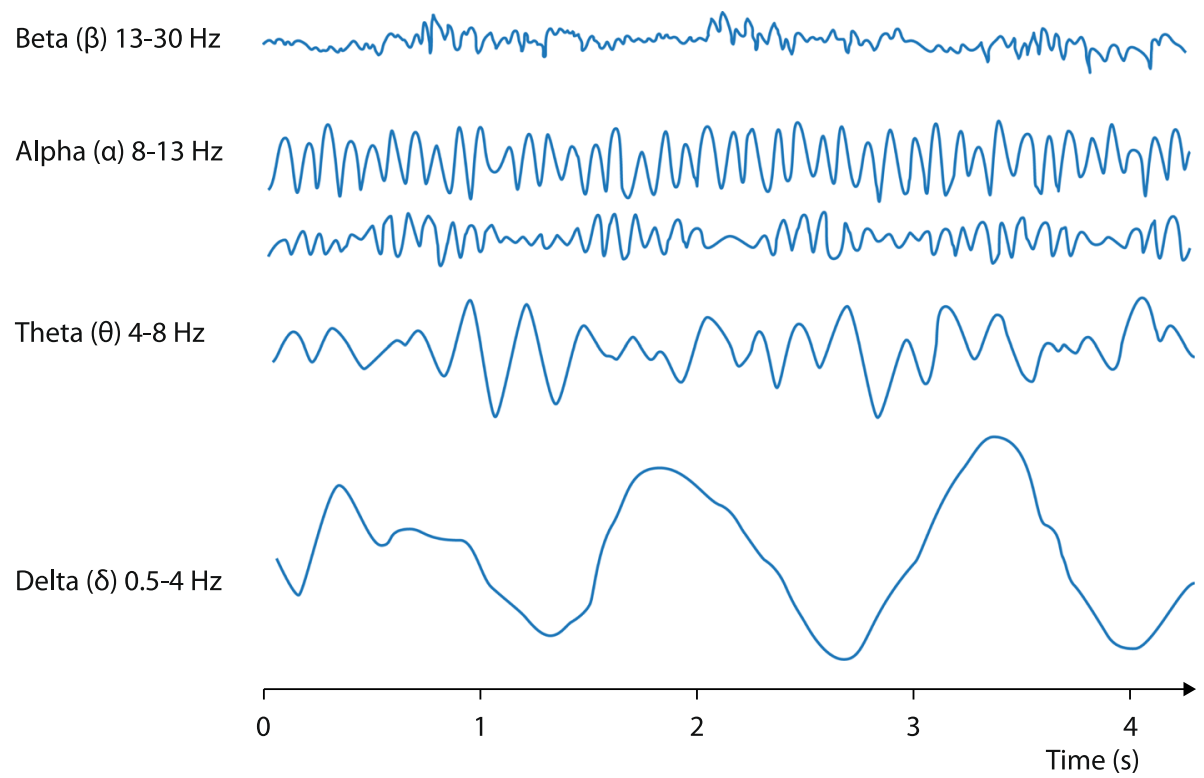

Fig. 3 EEG waveform frequency. Examples of traditional classification of alpha, beta, theta, delta EEG frequencies 


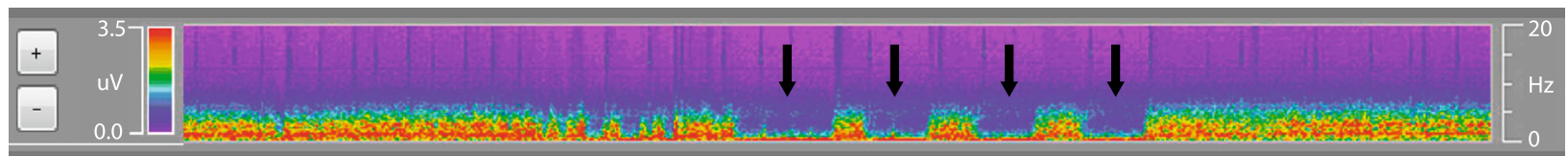

Fig. 4 Color density spectral array. In this frequency $(y)$ vs time(x) graph, each pixel shows the amplitude of EEG recording, according to its color (purple low, red high). The color range for the amplitude scale is shown on the left. Waveform frequency is displayed on the right. Phases of sudden reduction of faster frequencies during sedation can be easily recognized (arrows)

continuous monitoring. Sometimes, it is necessary to clean the skin or change the location of some electrodes to avoid scalp ulceration or infection.

\section{EEG Analysis}

EEGs may be categorized based on amplitude, frequency, symmetry and patterns. Although amplitude is measured quantitatively in microvolts, it is generally reported as low or high compared to a previously measured baseline. Amplitude has been somewhat arbitrarily divided into four frequency ranges (Fig. 3). From the highest to the lowest frequencies these waves are:

- Beta waves - frequency range $15-30 \mathrm{~Hz}$ and an amplitude of about $30 \mu \mathrm{v}$

- Alpha waves - frequency range 8-14 $\mathrm{Hz}$ and an amplitude of about 30-50 $\mu \mathrm{v}$
- Theta waves - frequency range 3-8 HZ and an amplitude of about 50-100 $\mu \mathrm{v}$

- Delta waves - frequency range $0.5-4 \mathrm{~Hz}$ and an amplitude of about $100-200 \mu v$.

Monitoring an ICU patient for several days generates gigabytes of data that, in their raw form, are nearly impossible for a neurophysiologist to review and to analyze at the bedside. Thus, several qEEG analyses have been implemented for use in cEEG monitoring. This involves fast Fourier transformation of raw EEG data performed in near real-time and subsequently displayed in compressed form. In this way, several hours of raw EEG recordings can be reduced to a single screen of time-frequency values.

Color density spectral array (DSA) is a graphical picture of the EEG that compresses hours of activity into time,

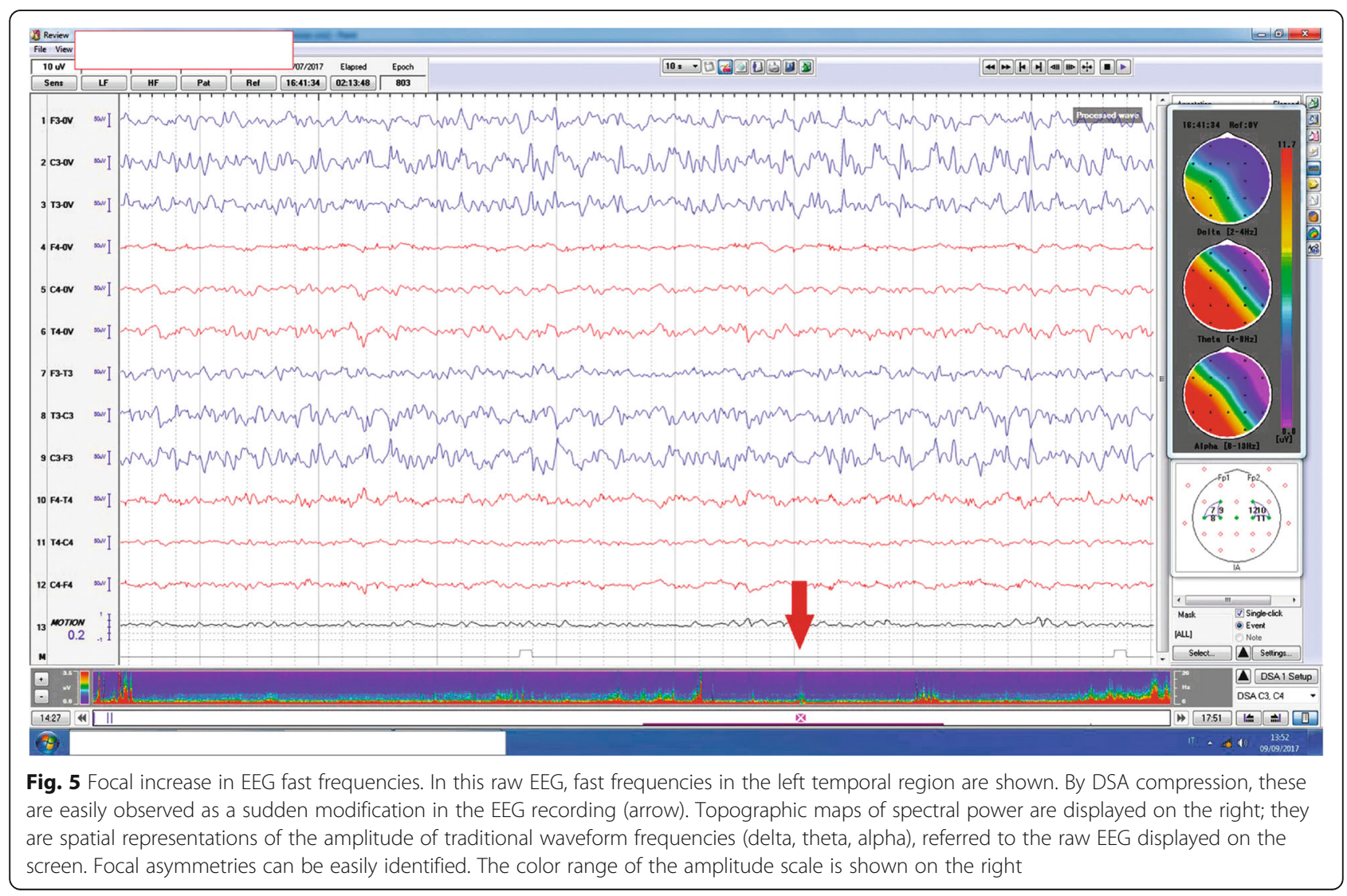


distribution of frequency, and power measurements. It is an X-Y graph, (frequency vs time), on which each pixel is colored according to the amplitude of the EEG recording. We can, therefore, easily observe amplitude in a particular range of frequency. Color DSA is very useful for rapid screening of EEG recordings, so as to identify significant abnormalities, such as seizures, effects of sedation, sleepwake cycle muscle artifacts (Fig. 4). In addition, it enables identification of gradual changes in the EEG trace, which are difficult to detect when reading EEG at a conventional review speed of $10 \mathrm{~s}$ per page.

Topographic maps of spectral power are graphical spatial representations, one for each classic frequency range (alpha, theta and delta), where each pixel is colored according to the amplitude of the signal, as with DSA. The maps enable rapid identification of asymmetries (Fig. 5).

Other graphical displays include ratios of power in certain bands over a broader spectrum of EEG power, envelope trends, amplitude-integrated EEG, and spectral edge displays. qEEG trends can be generated for individual EEG channels or combinations of channels to provide overviews of homologous left and right brain regions [38].

\section{Conclusion}

Use of cEEG has become more frequent over the last decade, and cEEG is considered one of the most important tools in multiparametric monitoring of neurocritical care patients. Since its introduction into clinical practice, several challenges have been solved, and international recommendations now use standardized terminology and indications and refer to the same technical issues. Nevertheless, clinical indications for cEEG are often not followed, and it still represents an underused tool.

One of the most important issues for its widespread diffusion is the 24/7 need for a technician to position and verify the electrodes, and a neurophysiologist to read the EEG. Several articles have shown that, after a relatively short period of training, ICU physicians and nurses can achieve an acceptable level in solving technical problems and identifying the main EEG patterns [39, 40]. This is an interesting challenge for neurointensivists. Further, at this time evidence that cEEG may improve patient outcome is still lacking. Nevertheless, its limited invasiveness, bedside availability and relatively low costs make cEEG an attractive tool with many potential areas of interest in the multiparametric monitoring of neurocritical care patients.

\section{Acknowledgements}

None

\section{Funding}

Publication costs were funded by "Catholic University School of Medicine".

\section{Availability of data and materials}

All data generated or analyzed during this study are included in this published article.

\section{Authors' contributions}

$\mathrm{AC}$ and IM analyzed and interpreted the patient data regarding CEEG in ICU. MA was a major contributor in revising the manuscript. All authors read and approved the final manuscript.

Ethics approval and consent to participate

Not applicable

Consent for publication

Not applicable

\section{Competing interests}

The authors declare that they have no competing interests.

\section{Publisher's Note}

Springer Nature remains neutral with regard to jurisdictional claims in published maps and institutional affiliations.

Published online: 20 March 2018

\section{References}

1. Nuwer MR. EEG and evoked potentials: monitoring cerebral function in the neurosurgical ICU. In: Martin NA, editor. Neurosurgical Intensive Care. Philadelphia: W.B. Saunders; 1994. p. 647-59.

2. Ney JP, van der Goes DN, Nuwer MR, Nelson L, Eccher MA. Continuous and routine EEG in intensive care: utilization and outcomes, United States 20052009. Neurology. 2013;81:2002-8.

3. Kinney MO, Kaplan PW. An update on the recognition and treatment of non-convulsive status epilepticus in the intensive care unit. Expert Rev Neurother. 2017;17:987-1002.

4. Herman ST, Abend N, Bleck TP. Consensus statement on continuous eeg in critically ill adults and children, Part I: Indications. J Clin Neurophysiol. 2015;32:87-95.

5. Claassen J, Taccone FS, Horn P, Holtkamp M, Stocchetti N, Oddo M Recommendations on the use of EEG monitoring in critically ill patients: consensus statement from the neurointensive care section of the ESICM. Intensive Care Med. 2013;39:1337-51.

6. André-Obadia N, Parain D, Szurhaj W. Continuous EEG monitoring in adults in the intensive care unit. Neurophysiol Clin. 2015;45:39-46.

7. Gavvala J, Abend N, LaRoche S, et al. Continuous EEG monitoring: a survey of neurophysiologists and neurointensivists. Epilepsia. 2014;55:1864-71.

8. Trinka E, Cock H, Hesdorffer D, et al. A definition and classification of status epilepticus - report of the ILAE task force on classification of status epilepticus. Epilepsia. 2015;56:1515-23.

9. Claassen J, Albers D, Schmidt JM, et al. Non convulsive seizures in subarachnoid hemorrhage link inflammation and outcome. Ann Neurol. 2014:75:771-81.

10. De Marchis GM, Pugin D, Meyers E, et al. Seizure burden in subarachnoid hemorrhage associated with functional and cognitive outcome. Neurology. 2016;86:253-60

11. Payne ET, Zhao XY, Frndova $H$, et al. Seizure burden is independently associated with short term outcome in critically ill children. Brain. 2014;137:1429-38.

12. DeLorenzo RJ, Waterhouse EJ, Towne AR, et al. Persistent nonconvulsive status epilepticus after the control of convulsive status epilepticus. Epilepsia. 1998;39:833-40

13. Laccheo I, Sonmezturk $H$, Bhatt $A B$, et al. Non-convulsive status epilepticus and non-convulsive seizures in neurological ICU patients. Neurocrit Care. 2015;22:202-11.

14. Towne AR, Waterhouse EJ, Boggs JG, et al. Prevalence of nonconvulsive status epilepticus in comatose patients. Neurology. 2000;54:340-5.

15. Oddo M, Carrera E, Claassen J, Mayer SA, Hirsch LJ. Continuous electroencephalography in the medical intensive care unit. Crit Care Med. 2009;37:2051-6.

16. Rodriguez Ruiz A, Vlachy J, Lee JW, et al. Association of periodic and rhythmic electroencephalographic patterns with seizures in critically ill patients. JAMA Neurol. 2017:74:181-8.

17. Vespa PM, McArthur DL, Xu Y, et al. Nonconvulsive seizures after traumatic brain injury are associated with hippocampal atrophy. Neurology. 2010;75:792-8 
18. Claassen J, Mayer SA, Kowalski RG, Emerson RG, Hirsch L. Detection of electrographic seizures with continuous EEG monitoring in critically ill patients. Neurology. 2004;62:1743-8.

19. Fogang $Y$, Legros B, Depondt C, et al. Yield of repeated intermittent EEG for seizure detection in critically ill adults. Clin Neurophysiol. 2017;47:5-12.

20. Westover MB, Shafi MM, Bianchi MT, et al. The probability of seizures during EEG monitoring in critically ill adults. Clin Neurophysiol. 2015;126:463-71.

21. Alvarez V, Sierra-Marcos A, Oddo M, Rossetti AO. Yield of intermittent versus continuous EEG in comatose survivors of cardiac arrest treated with hypothermia. Crit Care. 2013;17:R190.

22. Diedler J, Sykora M, Bast T, et al. Quantitative EEG correlates of low cerebral perfusion in severe stroke. Neurocrit Care. 2009;11:210-6.

23. Skordilis M, Rich N, Viloria A, et al. Processed electroencephalogram response of patients undergoing carotid endarterectomy: a pilot study. Ann Vasc Surg. 2011:25:909-12.

24. Mishra M, Banday M, Derakhshani R, Croom J, Camarata PJ. A quantitative EEG method for detecting post clamp changes during carotid endarterectomy. J Clin Monit Comput. 2011;25:295-308.

25. Gaspard N. Current clinical evidence supporting the use of continuous eeg monitoring for delayed cerebral ischemia detection. J Clin Neurophysiol. 2016;33:211-6.

26. Claassen J, Hirsch LJ, Kreiter KT, et al. Quantitative continuous EEG for detecting delayed cerebral ischemia in patients with poor-grade subarachnoid hemorrhage. Clin Neurophysiol. 2004:115:2699-710.

27. Rots ML, van Putten MJAM, Hoedemaekers CWE, Horn J. Continuous EEG monitoring for early detection of delayed cerebral ischemia in subarachnoid hemorrhage: a pilot study. Neurocrit Care. 2016;24:207-16.

28. Gollwitzer S, Groemer T, Rampp S, et al. Early prediction of delayed cerebral ischemia in subarachnoid hemorrhage based on quantitative EEG: a prospective study in adults. Clin Neurophysiol. 2015;126:1514-23.

29. Muniz CF, Shenoy AV, O'Connor KL, et al. Clinical development and implementation of an institutional guideline for prospective EEG monitoring and reporting of delayed cerebral ischemia. J Clin Neurophysiol. 2016;33:217-26.

30. Velly L, Pellegrini L, Bruder N. EEG en reanimation: quelles indications, quel materiel? Ann Fr Anesth Reanim. 2012;31:e145-53.

31. Sandroni C, Cariou A, Cavallaro F, et al. Prognostication in comatose survivors of cardiac arrest: an advisory statement from the European Resuscitation Council and the European Society of Intensive Care Medicine. Intensive Care Med. 2014;40:1816-31.

32. Westhall E, Rossetti AO, Van Rootselaar AF. Standardized EEG interpretation accurately predicts prognosis after cardiac arrest. Neurology. 2016;86:1482-90.

33. Claassen J, Hirsch LJ, Frontera JA, et al. Prognostic significance of continuous EEG monitoring in patients with poor-grade subarachnoid hemorrhage. Neurocrit Care. 2006;4:103-12.

34. American Clinical Neurophysiology Society. Guideline 1: Minimum technical requirements for performing clinical electroencephalography. J Clin Neurophysiol. 2006;23:86-91.

35. Vulliemoz S, Perrig S, Pellise D, et al. Imaging compatible electrodes for continuous electroencephalogram monitoring in the intensive care unit. J Clin Neurophysiol. 2009;26:236-43.

36. Young GB, Ives JR, Chapman MG, Mirsattari SM. A comparison of subdermal wire electrodes with collodion-applied disk electrodes in long-term EEG recordings in ICU. Clin Neurophysiol. 2006;117:1376-9.

37. Tanner AE, Sarkela MO, Virtanen J, et al. Application of subhairline EEG montage in intensive care unit: comparison with full montage. J Clin Neurophysiol. 2014;31:181-6.

38. Scheuer ML, Wilson SB. Data analysis for continuous EEG monitoring in the ICU: seeing the forest and the trees. J Clin Neurophysiol. 2004;21:353-78.

39. Dericioglu N, Yetim E, Bas DF, et al. Nonexpert use of quantitative EEG displays for seizure identification in the adult neuro-intensive care unit. Epilepsy Res. 2015;109:48-56.

40. Citerio G, Patruno A, Beretta S, et al. Implementation of continuous aEEG in two neurointensive care units by intensivists: a feasibility study. Intensive Care Med. 2017:43:1067-8 